\title{
Immune-Mediated Colitis
}

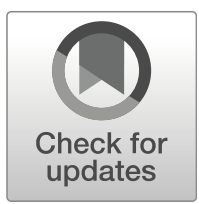

\section{Tara Menon, $M D^{*}$ \\ Anita Afzali, MD, MPH, FACG}

\author{
Address \\ "The Ohio State University Inflammatory Bowel Disease Center, Division of \\ Gastroenterology, Hepatology, and Nutrition, The Ohio State University Wexner \\ Medical Center, Columbus, OH, USA \\ Email: tara.menon@osumc.edu
}

Published online: 18 November 2019

(C) The Author(s) 2019. This article is an open access publication

This article is part of the Topical Collection on Gastroenterology for Geriatric Patients

Keywords Immune-mediated colitis · Immune checkpoint inhibitor · CTLA-4 · PD-L1 · PD-1 • Immune-related adverse events

\begin{abstract}
Purpose of review This review addresses our current knowledge of immune-mediated colitis (IMC) and offers a practical guide to its management.

Recent findings Due to the similarity in clinical, endoscopic, and histologic findings between IMC and inflammatory bowel disease (IBD), gastroenterologists have tailored their approach to IMC management to that of IBD.

Summary Immune checkpoint inhibitors (ICIs) are monoclonal antibodies that augment the T-cell anti-tumor response of the immune system and have demonstrated their importance in the treatment of a wide range of malignancies. With the growing benefits of ICIs, there are immune-related adverse events (irAEs) that mirror many known autoimmune diseases. Diarrhea and IMC are the most common and severe irAEs noted. No standardized guidelines exist in the management of these irAEs.
\end{abstract}

\section{Introduction}

Over the past several years, immune checkpoint inhibitors (ICIs) have had growing success in the treatment of a wide range of malignancies and have changed the treatment landscape in oncology. Cancer cells often use checkpoint molecules, such as cytotoxic $\mathrm{T}$ lymphocyte-associated antigen-4 (CTLA-4), programmed cell death protein-1 (PD-1), and programmed cell death ligand-1 (PD-L1), to evade attack from the immune system and allow for uninterrupted multiplication. ICIs inhibit these checkpoint pathways by blocking ligands or proteins that would otherwise lead to inactivation or death of anti-tumor T cells, which in turn allow $\mathrm{T}$ cells to destroy the cancer cells $[1 \bullet, 2]$.

The United States Food and Drug Administration (FDA) has approved several ICIs in the treatment of solid tumors and hematologic malignancies. Ipilimumab, an anti-CTLA-4 monoclonal antibody, was the first ICI approved for use in patients with malignant melanoma [3, $4 \bullet \bullet$. Pembrolizumab and nivolumab, agents that target 
PD-1, were subsequently approved in the treatment of melanoma. ICIs have now become the treatment of choice in several cancers including nonsmall cell lung cancer (NSCLC), Hodgkin lymphoma, renal cell carcinoma, hepatocellular carcinoma, head and neck squamous cancers, gastric adenocarcinoma, urothelial carcinoma, and mismatch repair-deficient/microsatellite instability-high solid tumors $[3,4 \bullet \bullet, 5]$. ICIs have been shown to improve outcomes and overall survival (OS) for oncology patients [6-8]. Many ICIs are currently in development, as the indications for these agents continue to expand. See Table 1 for a list of ICIs currently used in oncology.

ICIs work by inhibiting checkpoints, which results in widespread amplification of the immune response. Since this augmentation in the T-cell response is not tumorspecific, there is concern that this may lead to autoimmune manifestations or immunotoxicities, as normal tissue is often affected [9]. These immunotoxicities are also known as immune-related adverse events (irAEs). The clinical spectrum of irAEs is wide, with the potential to affect virtually any organ. The gastrointestinal tract is one of the most commonly affected systems and is often associated with the most serious irAEs [10,11]. Presentations range from self-limiting diarrhea to severe, lifethreatening immune-mediated colitis (IMC) $[6,10,11$, $12 \bullet, 13]$.

As indications for ICIs continue to grow, guidelines for the management of irAEs are needed. Since irAEs are essentially autoimmune diseases, we typically approach management with immunosuppression using corticosteroids or biologic medications (i.e., infliximab or vedolizumab) $[1 \bullet]$. The aims of this review are to summarize current knowledge regarding IMC and provide an overview of the management of this disease process.

\title{
Immune-related adverse events predict favorable response
}

Interestingly, development of irAEs is actually associated with increased efficacy of the ICI and improved oncologic outcomes $[1 \bullet, 14-21]$. Haratani and colleagues showed that the median progression-free survival was 9.2 months (95\% CI, 4.4 to not reached) in those who developed irAEs versus 4.8 months $(95 \% \mathrm{CI}$, 3.0 to 7.5 ) in those who did not develop irAEs. Moreover, multivariable analysis revealed that irAEs were positively associated with survival outcome [18].

Diarrhea is the most common gastrointestinal irAE and is an independent predictor of improved survival regardless of its related treatment [1•]. A study of

\section{Table 1. Immune checkpoint inhibitors}

\author{
Anti-CTLA-4 \\ Ipilimumab \\ Tremelimumab* \\ Anti-PD-1 \\ Pembrolizumab \\ Nivolumab \\ Cemiplimab \\ Pidilizumab* \\ Anti-PD-L1 \\ Atezolizumab \\ Avelumab \\ Durvalumab \\ *Currently not FDA approved
}




\section{Epidemiology}

39 patients who developed enterocolitis had significantly higher tumor response rates (36\% in multiple myeloma, 35\% in renal cell carcinoma) than those who did not experience this irAE (11\% in multiple myeloma and 3\% in renal cell carcinoma) [15]. However, another study of 298 patients with melanoma treated with ipilimumab found no improvement in OS in those who experienced any irAE compared with those who did not [22]. Another study also demonstrated that increased toxicity did not appear to correlate with improved response rates [23].

\section{Incidence}

\section{Incidence-immune-related adverse events overall}

Studies report an incidence as high as $90 \%$ for any grade irAEs due to ICI monotherapy $[6,24]$. A meta-analysis indicates an incidence of $72 \%(95 \% \mathrm{CI}$, 65-79\%) associated with CTLA-4 blockade [25] and 15 to 50\% in PD-1 or PDL1 blockade [17, 26-28].

Incidence and severity of irAEs is higher in patients on anti-CTLA-4/anti-PD1 combination therapy (44\%) than in those receiving anti-CTLA-4 (23-33\%) or anti-PD-1 ( $\leq 19 \%)$ monotherapy. Generally, the proportion of patients experiencing high-grade symptoms is greater with anti-CTLA-4 than anti-PD-1/ anti-PD-L1 agents $[2,29,30]$.

There are discordant studies on whether higher doses of these ICI agents lead to greater toxicities. An association is seen in CTLA- 4 blockade, in which a greater toxicity was noted at higher doses with agents such as ipilimumab $[6,15$, $24,31,32]$. In contrast, another study showed no increase in endoscopic ulcerations or the need for immunosuppression in patients who received higher doses of anti-CTLA-4 agents [33•]. This dose-toxicity relationship is not seen with anti-PD-1 or anti-PD-L1 therapy [34, 35].

\section{Incidence-gastrointestinal immune-related adverse events}

Diarrhea is a common irAE in immunotherapy with an incidence of 35\% for anti-CTLA-4 agents, $20 \%$ for anti-PD-1 agents, and $44 \%$ for combination therapy [29, 36-38]. Gupta and colleagues noted watery diarrhea in $27-54 \%$ of those on anti-CTLA-4 therapy, such as ipilimumab and tremelimumab, with symptoms occurring within days to weeks of therapy initiation [39].

In a study of 198 patients with metastatic melanoma or renal cell carcinoma treated with CTLA-4 blocker ipilimumab, 21\% developed enterocolitis, making it the most common major toxicity [15]. Gupta and colleagues found that diffuse acute and chronic colitis was the most common finding on endoscopy $(8-22 \%)$ [39]. In a meta-analysis, the incidence of severe IMC was $9.1 \%$ with CTLA-4 monotherapy, 1.3\% with anti-PD-1/anti-PD-L1 therapy, and 13.6\% with combined therapy [40]. Incidence of IMC in anti-CTLA-4 treatment was noted to be approximately 4 to $5 \%[36,41,42]$ and 1 to $4 \%$ in anti-PD-1 and anti-PD-L1 therapy $[17,20,21,43,44]$.

Diarrhea and colitis have been found to be the most common reasons for ICI therapy discontinuation $[22,29]$. The prevalence of gastrointestinal irAEs differed, being less common in anti-PD-1 or anti-PD-L1 compared to antiCTLA-4 agents [2, 6, 17, 29]. 


\section{Nonsteroidal anti-inflammatory drugs}

Marthey and colleagues demonstrated that among the 35 study participants who developed IMC, nonsteroidal anti-inflammatory drugs (NSAIDS) were more frequently used in those who developed enterocolitis than in those who did not (31 vs $5 \%, p=0.003$ ) [33•]. This is similar to inflammatory bowel disease (IBD) and the association of NSAIDS with increased risk for IBD flare [45].

\section{Medical history of autoimmune disease}

Patients who have active underlying autoimmune disease are at risk for worsening of their autoimmune diseases while on ICI immunotherapy. In one study of patients with NSCLC on ICI immunotherapy, immunosuppression was required in 54\% with rheumatoid arthritis, 38\% with psoriasis, and $100 \%$ with polymyalgia rheumatic, Sjogren's syndrome, or immune thrombocytopenic purpura [46]. Two of the six patients with ulcerative colitis (UC) or Crohn's disease (CD) had ipilimumab-induced colitis or relapse of their IBD [47]. Very few data are available on the risk of IMC in patients with underlying IBD, as it is difficult to differentiate the two entities.

\section{Medical history of immune-related adverse events}

A few studies have shown that those who have had irAEs with one ICI agent are at increased risk for developing irAEs following initiation of an alternate ICI [23, 46]. Conversely, other studies have shown that patients with significant diarrhea or IMC during ipilimumab treatment have subsequently been treated with anti-PD-1 or anti-PD-L1 immunotherapy without developing recurrence of diarrhea or IMC $[2,42]$.

\section{Combination therapy with two immune checkpoint inhibitors}

In the treatment of metastatic melanoma, studies show that combination therapy with an anti-PD-1 and an anti-CTLA- 4 agent results in longer patient OS [48]. As previously described, the incidence of irAEs on combination therapy is higher than in those on monotherapy [29, 48-50]. Although irAEs have been known to develop in the first few weeks or months of immunotherapy, those on combination therapy may be at risk of developing irAEs over a longer period of time [22]. Patients treated with combination CTLA- 4 and PD- 1 blockade develop more frequent and severe toxicities characteristic of both single agents, but do not appear to develop any irAEs that are unique to combination therapy [51].

\section{Sequential therapy}

Two case series report prolonged, difficult-to -treat IMC in patients who were treated with CTLA-4 blockade after anti-PD-1 antibody treatment $[52,53]$. This was also seen in a subsequent study, where over one third of patients experienced IMC when treated with anti-PD-1 therapy followed by ipilimumab [23]. 
Table 2. Immune checkpoint inhibitor severity from the Common Terminology Criteria for Adverse Events version 5.0 (CTCAE v5.0)

\begin{tabular}{lll}
\hline & Diarrhea & Colitis \\
Grade 1 & $<4$ stools/day over baseline & Asymptomatic \\
Grade 2 & $4-6$ stools/day over baseline & Abdominal pain, blood, and/or mucus in stool \\
Grade 3 & $\geq 7$ stools/day over baseline & Severe abdominal pain, fever, and/or peritoneal signs \\
Grade 4 & Life-threatening & Life-threatening \\
& - Hemodynamics & - Perforation \\
& - Electrolyte abnormalities & - Ischemia \\
& & - Necrosis \\
Grade 5 & Death & - Bleeding \\
Adapted from the Common Terminology Criteria for Adverse Events version 5.0 from the National Cancer Institute [86]
\end{tabular}

\section{Diagnosis}

\section{Clinical presentation}

GI symptoms are less common with nivolumab than ipilimumab [29], possibly because PD-1 or PD-L1 seems to play less of a role in gut homeostasis than CTLA-4 [24, 29, 36, 54].

In those with IMC, the most common presenting symptoms are diarrhea (92\%), abdominal pain (82\%), hematochezia (64\%), fever (46\%), and vomiting (36\%) [33•]. The median weight loss is approximately $8 \%$ of total body weight [33•]. The rate of bowel perforation is less than $1 \%$ and is considered uncommon [36, 54].

Onset of symptoms can occur at any point during the treatment course of anti-CTLA-4 immunotherapy, but frequently occur between the second and third doses $[9,15,25,55]$. Colitis related to anti-PD-1/PD-L1 immunotherapy can also present at any point in the course, and some patients present even years into therapy, raising the hypothesis of ICI therapy being a potential trigger for underlying IBD [10].

Lord and colleagues demonstrated that symptoms may persist for several months after the last ICI dose. They also noted that the length of symptoms exceeded 130 days in two of their patients, which is greater than ten times the 12.5-day half-life of ipilimumab. This suggests that ICI therapy has a long-term effect on the immune system rather than checkpoint inhibition in the immune cascade simply at the time of treatment [55].

Case studies have also reported pancreatitis, hepatotoxicity, gastritis, and celiac disease due to ICIs [9, 56-58].

\section{Grading}

Classification and degree of severity are most commonly based on the National Cancer Institute (NCI) Common Terminology Criteria for Adverse Events (CTCAE), version 5.0 [86]. Unfortunately, this grading is arbitrary, as it is based on patient recall of symptoms and not on objective assessment of disease 
activity. Consequently, it does not necessarily reflect the severity of the immunotoxicity [22]. Finally, there is poor correlation between grade of diarrhea and endoscopic or histological features for severity $[16,38]$. See Table 2 for the CTCAE grading system.

Since there is poor correlation between grade of diarrhea and endoscopic or histological features for severity, endoscopic evaluation should be strongly considered in those with symptoms more severe than CTCAE grade 1 diarrhea $[16,38]$. In fact, the gold standard for diagnosis of IMC is endoscopy to better evaluate the mucosal extent and severity of disease. A colonoscopy with terminal ileum intubation, radiologic cross-sectional imaging, and/or video capsule endoscopy may be needed if isolated enteritis is suspected, as sometimes seen in anti-PD-1/anti-PD-L1 immunotherapy [33•].

Mild colitis is the most common finding in the colon and presents with diarrhea, affecting nearly half of the patients on high dose or combination therapy $[24,29,36,54]$. Severe colitis occurs less frequently, but can be lifethreatening $[24,29,36,54]$. Many of the endoscopic and histologic features of IMC are similar to features seen in IBD [41]. Ipilimumab-induced colitis most closely resembles ulcerative pancolitis, which is seen in $66 \%$ of patients with IMC [33•]. Inflammation presents endoscopically as diffuse erythema, friability, shallow ulcerations, and edematous walls from cecum to anus [33•]. In contrast, segmental disease, as is often found in Crohn's disease, is less common and noted in $35 \%$ of individuals with IMC [33•]. Stricturing or fistulizing disease that is associated with Crohn's disease is not typically seen with IMC. Although most patients develop colitis alone, approximately a quarter of patients with colonic involvement also have diffuse enteritis [15].

In studies that evaluated anti-CTLA-4-associated enterocolitis, the rectum and sigmoid were involved $97 \%$ of the time [33•]. Therefore, a flexible sigmoidoscopy may suffice as the diagnostic tool in the vast majority of cases and a full colonoscopy may not be needed $[33 \bullet, 59]$.

Those with ulcerations on initial endoscopy had a significantly higher rate of high-grade diarrhea than those without ulcers (100 vs $79 \%$, $p=0.033$ ) [16]. There is no specific grading scale used for the endoscopic findings in IMC, but the Mayo endoscopic score for ulcerative colitis or the Simple Endoscopic Score for Crohn's disease (SES-CD) may be used [38].

A relatively high frequency of patients treated with PD-1/PD-L1 blockade develop low-grade diarrhea, but this rarely progresses to severe colitis [29, 43, 60-63]. Although severe colonic inflammation is much less common with PD1/PD-L1 blockade than with anti-CTLA-4 immunotherapy, pneumonitis is more strongly associated with inhibition of the PD-1/PD-L1 pathway, emphasizing the different regulatory roles of these receptors within distinct tissues [41].

IMC due to CTLA-4 inhibitors can have lymphocytic and/or neutrophilic infiltrates as well as increased numbers of apoptotic cells on histology [15, 33•, 38]. Neutrophilic inflammation was predominantly characterized by cryptitis with one-third showing crypt abscesses [15]. Lymphocytic predominance 
demonstrating crypts and prominent eosinophils is less commonly seen. Although these can be present, it is less likely to see chronic architectural changes or granulomas. This is in contrast to IBD, in which chronic crypt architectural changes are often seen due to the chronicity of disease.

Notably, anti-PD-1/PD-L1 immunotherapy induces small intestinal and colonic inflammation that is clinically distinct from colitis induced by ipilimumab. Although these patients may develop low-grade diarrhea from isolated enteritis or colitis, histologic changes seem to resemble microscopic colitis [29, 43, 60-63].

Laboratory studies

Anemia, hypoalbuminemia, and elevated inflammatory markers, such as Creactive protein and fecal calprotectin, are seen in IMC. As in IBD, these findings are nonspecific and may vary between individuals [33•]. Fecal calprotectin may be helpful in monitoring IMC disease activity [9, 64•, 65-67].

Radiologic studies

There are no distinct radiographic findings noted in IMC [68]. If there is concern for abscess or perforation, then cross-sectional imaging should be pursued [41]. If there is concern for enteritis, then enterography can be considered.

\section{Management}

There are no standardized guidelines in the management of IMC, but most recommendations are largely based on degree of severity. Infectious etiology for symptoms should be considered first. Stool studies for Clostridium difficile and other bacterial pathogens are recommended. Stool parasitic testing should be based on risk factors and provider discretion $[10,69]$. Preliminary blood work including complete blood count, comprehensive metabolic panel, thyroid stimulating hormone, celiac disease serology, erythrocytes sedimentation rate, and C-reactive protein should be performed. Calprotectin may be helpful to monitor treatment response [9]. Other screening labs to obtain include hepatitis A and B serology, as well as tuberculosis testing, in preparation for possible initiation of biologic therapy if not responsive to corticosteroids.

For severe disease, corticosteroids should be used initially. If refractory to corticosteroids, biologic therapy with medications such as infliximab, an antitumor necrosis factor (TNF), or vedolizumab, an anti-integrin, should be considered. Wang and colleagues demonstrated that $56 \%$ of patients responded to corticosteroids alone, and $42 \%$ were corticosteroid-refractory but did respond to infliximab [16]. Approximately one third to two third of colitis cases have recurrence after corticosteroid taper or are refractory to corticosteroids [10, $15,33 \bullet, 59]$. Infliximab remains the most studied second-line agent $[6,70]$. In most cases, only one to three doses $[10,15]$ are needed to improve symptoms. It is recommended that endoscopic evaluation with biopsies be performed to ensure mucosal and endoscopic healing.

In one study, patients treated with infliximab had a shorter time to symptom resolution. Resolution of diarrhea was 3 days in those who received corticosteroids followed by infliximab versus 9 days in those who received corticosteroids alone $(p<0.001)$. In those with colitis, symptom recovery was 4 days in 


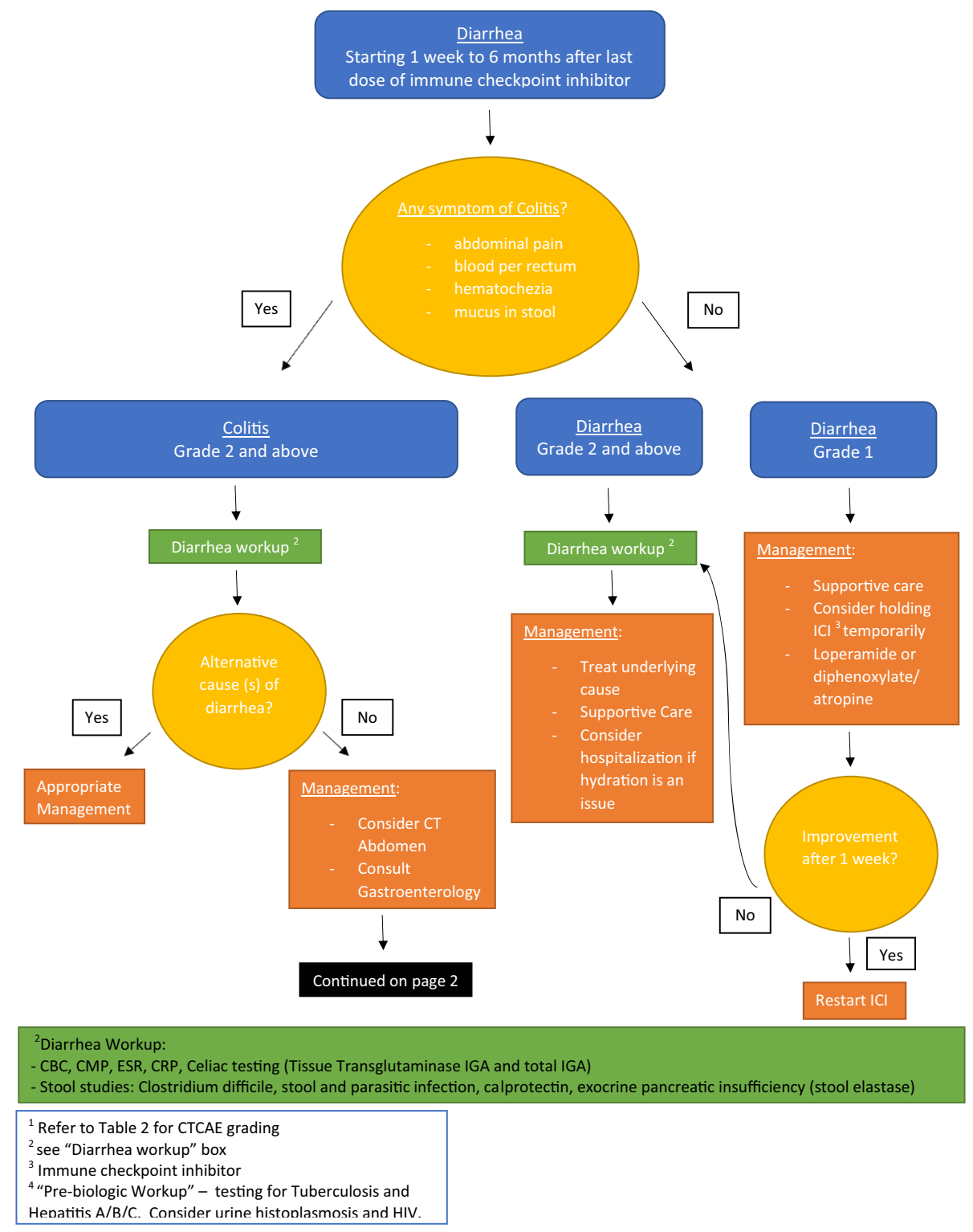

Fig. 1. Approach to diarrhea in the setting of immune checkpoint inhibitors based on MD Anderson Cancer Center's "Evaluation and Management of Suspected Immune-Mediated Colitis/Diarrhea" [73••].

those who received corticosteroids and infliximab and 13 days in those who received only corticosteroids $(p<0.001)$. This was despite the infliximab group having more severe irAEs initially. There were also shortened length of hospital stay and improved quality of life, as well as greater avoidance of high-dose corticosteroids and their associated adverse effects [70]. Lastly, those who received two or less infusions of infliximab had less histologic remission and higher fecal calprotectin levels than those who received three or more infusions $[71 \bullet]$.

Although current recommendations advise biologic use in patients who are refractory to corticosteroids, there are no recommendations to guide the use of 


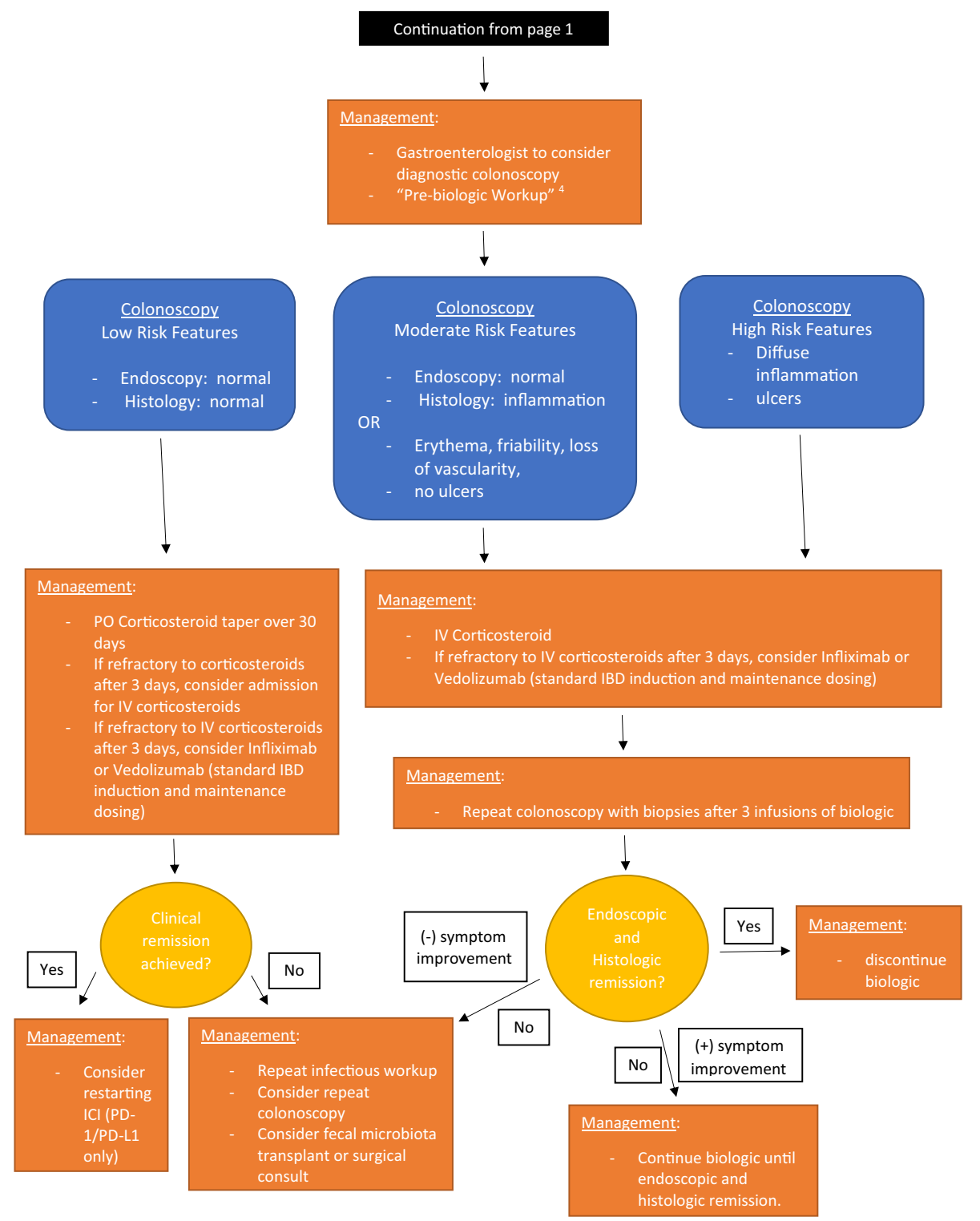

Fig. 1. (continued)

the biologic once it is initiated $[4 \bullet \bullet, 6,10,72]$. See Fig. 1 for a basic approach to diarrhea and IMC modified from hospital guidelines developed at MD Ander-

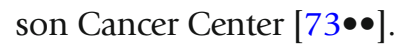

The American Society of Clinical Oncology (ASCO) and the National Comprehensive Cancer Network ( $\mathrm{NCCN}$ ) guidelines also recommend to first deter-

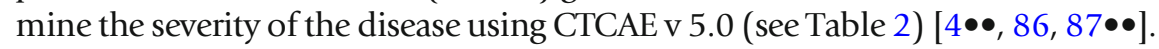
Symptomatic treatment with anti-diarrheal medications is recommended for grade 1 . Treatment with ICI can typically be continued. Careful monitoring and supportive care is recommended and the IMC is usually self-limiting.

If the patient has a grade 2 toxicity, ASCO recommends that the ICI be held until symptoms improve to grade 1 or less. One can also consider permanently 
discontinuing anti-CTLA-4 agent and starting anti-PD-1 or anti-PD-L1 therapy if the patient's condition improves off of an ICI. A gastroenterology consult should be placed for further diagnosis and management. A flexible sigmoidoscopy or colonoscopy and/or esophagogastroduodenoscopy may be pursued. Immunosuppressive maintenance with $1 \mathrm{mg} / \mathrm{kg} /$ day of prednisone (or equivalent) may be initiated. A long taper is recommended over 4-6 weeks once symptoms have improved to a grade 1 or less $[4 \bullet \bullet, 6,9]$.

For grade 3 toxicities, permanently discontinuing anti-CTLA- 4 agents is recommended. The patient may require medical attention for treatment of dehydration or any electrolyte imbalances. Corticosteroids should be initiated (initial dose of 1 to $2 \mathrm{mg} / \mathrm{kg} /$ day prednisone or equivalent). If symptoms persist $\geq 3$ to 5 days or recur after initial improvement, intravenous corticosteroids or biologic therapy with infliximab may be initiated. A repeat colonoscopy in some cases may be indicated, particularly to rule out concomitant opportunistic infections to treatment refractory disease $[4 \bullet \bullet, 6,9,10,22,69]$. If symptoms recover to grade 1 or less, patients may be started on anti-PD-1/antiPD-L1 agents if indicated and infliximab discontinued.

For grade 4 toxicities, the ICI treatment should be permanently discontinued. The patient may require a hospital admission to closely monitor and treat the diarrhea and its complications. Gastroenterology consultation is needed to further guide management. Intravenous corticosteroids should be administered and not changed to oral formulations until symptoms start to improve. It is recommended that infliximab $5-10 \mathrm{mg} / \mathrm{kg}$ therapy be initiated and continued in patients who do not respond to corticosteroids within 23 days. Endoscopy is recommended for refractory or severe illness [9].

\section{Response to therapy}

In anti-CTLA-4 enterocolitis, $37 \%$ of the 39 patients achieved complete remission on corticosteroids. Of the 12 patients who required infliximab, ten (83\%) responded [33•].

Overall, one third to two thirds of patients either do not respond to highdose intravenous corticosteroids or have a relapse requiring an increase in the corticosteroid dosage during the course of steroid tapering $[5,7]$. These patients usually have an excellent response to infliximab (dose $5 \mathrm{mg} / \mathrm{kg}$ ) $[10,15,22$, $33 \bullet, 59,74]$.

It is recommended to assess for endoscopic and histologic remission and not base decision-making on clinical symptoms alone, as there is a known discrepancy between symptoms and mucosal healing, as also seen in IBD [71 $\bullet$.

Although biologic drug trough levels for assessment of therapeutic drug monitoring can be used to assist in decision-making, target drug goal levels are not well understood in IMC. However, if adequate drug levels cannot be reached and the patient has not achieved a response, switch in biologic class of therapy to an alternative mechanism of action should be considered. 
currently no predictors for response. It is possible that these patients have underlying or unmasked IBD that has been triggered by the ICI [9].

\section{History of IBD}

Those patients who have a history of IBD, which was quiescent at the time of ICI initiation, may be more refractory to treatment if they develop IMC. ICIs should be avoided in patients with active IBD in the setting of malignancy [47]. Very few studies are available on the risk of IMC in patients with CD or UC, as these entities are difficult to differentiate.

\section{Infections}

As in IBD, infections such as CMV and HSV should be excluded for refractory disease. Endoscopy with biopsies is the recommended approach.

\section{Vedolizumab}

Vedolizumab, an anti-integrin, is being explored as an effective alternative to anti-TNF therapy in the setting of IMC, as either a first-line biologic after corticosteroid failure or as a second-line biologic in anti-TNF primary nonresponders [64•]. Vedolizumab is gut-selective and may have a better safety profile $[60,64 \bullet, 74-76]$.

Vedolizumab can also be considered in the treatment of IMC in patients with a contraindication to anti-TNF agents, such as those with New York Heart Association (NYHA) Functional Classification greater than 3, latent tuberculosis, or multiple sclerosis [64•].

Abu-Sbeih and colleagues [64•] compared the response in patients who failed both corticosteroids and infliximab before trialing vedolizumab versus those who failed just corticosteroids prior to initiation of vedolizumab. There was a shorter mean duration of corticosteroid use in the second group ( $85 \mathrm{vs}$ 131 days). Vedolizumab led to clinical remission in 84\% patients with corticosteroid-refractory ICI colitis and endoscopic remission in 54\% of patients. The mean duration of improvement of symptoms was 5 days after the initial dose of vedolizumab. In a case series of seven patients, all but one patient experienced corticosteroid-free IMC remission with normalized fecal calprotectin after vedolizumab therapy. This was achieved after a median of 56 days from vedolizumab initiation, and no adverse effects from biologic treatment were noted [75].

As previously demonstrated among IBD patients, a better response rate is seen in anti-TNF naïve patients treated with vedolizumab as first-line therapy. Those receiving a longer duration of corticosteroid therapy were also found to have lower responses to vedolizumab [64•].

\section{Fecal microbiota transplant}

There is increased bacterial diversity of the microbiome in those who did not develop IMC with ipilimumab immunotherapy [77-80]. Studies are currently under investigation for the potential role of fecal microbiota transplant (FMT) in IMC. In one case series, FMT performed on biologic-refractory IMC patients did have clinical, endoscopic, and histologic improvement [81]. 


\section{Colectomy}

In a study of 39 patients with anti-CTLA-4-mediated colitis, six patients required colectomy (five due to perforation and one due to toxic megacolon) [33•]. If surgery is needed, subtotal colectomy, as opposed to a segmental colectomy, is recommended as pancolitis is the most common presentation [33•].

\section{Prophylaxis}

Prophylactic treatment with corticosteroids, including budesonide, on patients undergoing treatment with ICIs did not prevent the development of diarrhea or colitis. Furthermore, it is not known if taking corticosteroids or infliximab concomitantly with the ICI will prevent IMC [33•, 42].

\section{Prognosis/follow-up}

\section{Recurrence}

During a mean follow-up of 19 months, 19 of 53 patients (36\%) experienced recurrent diarrhea despite successful initial treatment with immunosuppression. Three were successfully treated with anti-diarrheal medications, ten needed a repeat course of corticosteroids, and six ultimately required infliximab maintenance therapy. The initial grade of diarrhea or colitis, presence of endoscopic or histologic inflammation, requirement of infliximab for initial treatment, or the duration of corticosteroid therapy was not associated with recurrence [16]. Based on these findings, it is not possible to predict which patients will experience recurrence and close follow-up is recommended even when a patient is initially in remission.

\section{Immunosuppression and efficacy}

There is a concern for tumor progression or attenuated response to ICIs if immunosuppression is needed with corticosteroids or biologics in the treatment of irAEs. Immunosuppressants work to suppress T-cell activation, which in theory, could negate the benefits of checkpoint inhibition. However, published findings do not support this theory with nivolumab, ipilimumab, or corticosteroids [14, 15, 17, 22, 82-85]. Of 576 patients with advanced melanoma evaluated in four clinical trials, Weber and colleagues found that in the $24 \%$ who received immunosuppressive therapy for irAEs, there was no significant difference in objective treatment response rates or the rates and effectiveness of the anti-cancer tumor response [17].

Those who develop severe irAEs do trend toward increased survival. However, the possibility of increased overall survival with corticosteroid-sparing treatments is unknown. No differences were found in overall survival between patients who received steroids without infliximab and those who received steroids with infliximab $(p=0.768)[1 \bullet]$. However, available studies investigating the effect of corticosteroid sparing treatments on overall survival are sparse.

\section{Question of progression to inflammatory bowel disease}

Studies have shown persistence of endoscopic and histologic inflammation several months after onset of treatment for IMC, with or without clinical symptoms. This observation stands true in patients with IBD, where it takes 
longer for histologic and endoscopic features to resolve when compared with clinical symptoms alone. It is unclear if this signifies a progression toward chronic inflammatory bowel disease $[33 \bullet, 64 \bullet]$.

\section{Reintroduction of checkpoint inhibitors after treatment for immune-mediated colitis}

Marthey and colleagues [33•] noted that four of the six patients who had an additional infusion of ipilimumab after IMC remission experienced a relapse. Three of these four patients required a repeat corticosteroid course with eventual achievement of remission. The fourth patient was refractory to the corticosteroid course and required an infliximab infusion. There is a high risk of relapse in those with IMC history who undergo reintroduction of a checkpoint inhibitor [33•]. Risks and benefits of retreatment with anti-CTLA-4 medications should therefore be closely weighed with the patient and oncology team.

\section{Infection risk with immunosuppression}

In a retrospective, single-center study, $37 \%$ of the 79 patients developed IMC or diarrhea and were given corticosteroids with or without biologic therapy. Duration of corticosteroid use in patients who developed infections was longer than in those who did not develop infections ( 80.4 vs 46.7 days; $p=0.063$ ). The median length of steroid treatment in patients who developed an infection was 60 days and only 34 days in patients who did not. No other significant differences in terms of cancer stage, malignancy type, ICI agent, diarrhea grade, or colitis grade were observed between patients who did or did not develop infections $[1 \bullet]$. These findings again suggest early initiation of corticosteroidsparing treatment.

\section{Conclusion}

ICIs have revolutionized the field of oncology and have become the standard of care for a wide range of malignancies. Despite important clinical benefits, these medications can cause irAEs, including IMC, which closely resembles and appears to be an accelerated form of IBD. To further prevent complications, prompt recognition of corticosteroid failure and subsequent initiation of biologics is imperative. Infliximab is most commonly recommended, but early studies show promise with vedolizumab therapy as well. Resumption of ICIs should be approached cautiously, as there is a high likelihood of relapse. A multidisciplinary group consisting of gastroenterologists and oncologists is necessary to provide comprehensive care and to ensure a methodical approach to this emerging disease spectrum.

\section{Author contributions}

MT: performed the research and wrote the paper. AA: contributed toward critical revision and intellectual content. 


\section{Compliance with Ethical Standards}

\section{Conflict of Interest}

Tara Menon declares that she has no conflict of interest. Anita Afzali is a consultant for Abbvie, UCB, Takeda, Pfizer, Janssen, and Celgene; and a board member and founder of IBD Horizons ${ }^{\circledR}$ and MyIBD.com.

\section{Human and Animal Rights and Informed Consent}

This article does not contain any studies with human or animal subjects performed by any of the authors.

Open Access This article is distributed under the terms of the Creative Commons Attribution 4.0 International License (http://creativecommons.org/licenses/by/4.0/), which permits unrestricted use, distribution, and reproduction in any medium, provided you give appropriate credit to the original author(s) and the source, provide a link to the Creative Commons license, and indicate if changes were made.

\section{References and Recommended Reading}

Papers of particular interest, published recently, have been highlighted as:

- Of importance

- Of major importance

1. Wang Y, Abu-Sbeih H, Mao E, et al. Immune-checkpoint inhibitor-induced diarrhea and colitis in patients with advanced malignancies: retrospective review at MD Anderson. J Immunother Cancer. 2018;6:37. https://doi. org/10.1186/s40425-018-0346-6

This study examines the link between immune-related adverse events and improved oncologic outcomes in patients on immunotherapy.

2. Kumar V, Chaudhary N, Garg M, et al. Current diagnosis and management of immune related adverse events (irAEs) induced by immune checkpoint inhibitor therapy. Front Pharmacol. 2017;8:49. https://doi. org/10.3389/fpharm.2017.00049.

3. Postow M, Wolchok J. Toxicities associated with checkpoint inhibitor immunotherapy. In: Atkins MB, editor. UpToDate. Waltham: UpToDate; 2018.

4.• Brahmer JR, Lacchetti C, Schneider BJ, et al. Management of immune-related adverse events in patients treated with immune checkpoint inhibitor therapy: American Society of Clinical Oncology Clinical Practice Guideline. J Clin Oncol. 2018;36(17):1714-68. https://doi.org/10.1200/JCO.2017.77.6385

This study provides guidance on the management of immunerelated adverse events in patients on immunotherapy.

5. Overman MJ, Lonardi S, Wang KYM, et al. Durable clinical benefit with nivolumab plus ipilimumab in DNA mismatch repair- deficient/microsatellite instability-high metastatic colorectal cancer. J Clin Oncol. 2018;36(8):773-9. https://doi.org/10.1200/ JCO.2017.76.9901.

6. Puzanov I, Diab A, Abdallah K, Bingham CO 3rd, Brogdon C, Dadu R, et al. Managing toxicities associated with immune checkpoint inhibitors: consensus recommendations from the Society for Immunotherapy of Cancer (SITC) toxicity management working group. J Immunother Cancer. 2017;5:95. https://doi.org/10.1186/s40425-017-0300-z.

7. Hoos A. Development of immune-oncology drugs-from CTLA4 to PD1 to next generations. Nat Rev Drug Discov. 2016;14(4):235-47. https://doi.org/ 10.1038/nrd.2015.35.

8. Papaioannou NE, Ourania V, Vitsos P, et al. Harnessing the immune system to improve cancer therapy. Ann Transl Med. 2016;4(14):261.

9. Weber JS, Kahler KC, Hauschild A, et al. Management of immune-related adverse events and kinetics of response with ipilimumab. J Clin Oncol. 2012;30(21):2691-7.

10. Haanen J, Carbonnel F, Robert C, Kerr KM, Peters S, Larkin J, et al. Management of toxicities from immunotherapy: ESMO clinical practice guidelines for diagnosis, treatment and follow-up. Ann Oncol. 2018;28:iv119-42. https://doi.org/10.1093/annonc/ $\operatorname{mdx} 225$.

11. Haanen J, Carbonnel F, Robert C, et al. Management of toxicities from immunotherapy: ESMO Clinical Practice Guidelines for diagnosis, treatment, and follow up. Ann Oncol Off J Euro Soc Med Oncol. 2017;28:iv119iv42.

12. Abu-Sbeih H, Ali FS, Luo W, et al. Importance of endoscopic and histologic evaluation in the management of immune checkpoint inhibitor-induced colitis. J Immunother Cancer. 2018;6(1):95. https://doi.org/10. 1186/s40425-018-0411-1 
This study highlights endoscopic and histologic features of immune-mediated colitis and their association with disease outcomes.

13. Michot JM, Bigenwald C, Champiat S, et al. Immunerelated adverse events with immune checkpoint blockade: a comprehensive review. Eur J Cancer. 2016;54:139-48.

14. Downey SG, Klapper JA, Smith FO, Yang JC, Sherry RM, Royal RE, et al. Prognostic factors related to clinical response in patients with metastatic melanoma treated by CTL-associated antigen-4 blockade. Clin Cancer Res. 2007;13:6681-8. https://doi.org/10.1158/1078-0432. CCR-07-0187.

15. Beck KE, Blansfield JA, Tran KQ, Feldman AL, Hughes MS, Royal RE, et al. Enterocolitis in patients with cancer after antibody blockade of cytotoxic T-lymphocyteassociated antigen 4. J Clin Oncol. 2006;24:2283-9. https://doi.org/10.1200/JCO.2005.04.5716.

16. Wang Y, Abu-Sbeih H, Mao E, et al. Endoscopic and histologic features of immune checkpoint inhibitorrelated colitis. Inflamm Bowel Dis. 2018;24:1695-705. https://doi.org/10.1093/ibd/izy104.

17. Weber JS, Hodi FS, Wolchok JD, Topalian SL, Schadendorf D, Larkin J, et al. Safety profile of nivolumab monotherapy: a pooled analysis of patients with advanced melanoma. J Clin Oncol.

2017;35(7):785-92.

18. Haratani K, Hayashi H, Chiba Y, Kudo K, Yonesaka K, Kato $\mathrm{R}$, et al. Association of immune related adverse events over a period of 6 weeks is associated with nivolumab efficacy in patients with non-small cell lung cancer. JAMA Oncol. 2018;4(3):374-8. https://doi. org/10.1001/jamaoncol.2017.2925.

19. Freeman-Keller M, Kim Y, Cronin H, Richards A, Gibney G, Weber JS. Nivolumab in resectable and unresectable metastatic melanoma: characteristics of immune related adverse events and associate with outcomes. Clin Cancer Res. 2016;22(4):886-94. https://doi.org/10.1158/1078-0432.CCR-15-1136.

20. Hamid O, Robert C, Daud A, et al. Safety and tumor responses with lambrolizumab (anti-PD-1) in melanoma. N Engl J Med. 2013;369:134-44.

21. Sarnaik AA, Yu B, Yu D, et al. Extended dose ipilimumab with a peptide vaccine: immune correlates associated with clinical benefit in patients with resected high-risk stage IIIC/IV melanoma. Clin Can Res An Off J Am Associ Cancer Rese. 2011;17:896-906.

22. Horvat TZ, Adel NG, Dang T-O, Momtaz P, Postow MA, Callahan MK, et al. Immune-related adverse events, need for systemic immunosuppression, and effects on survival and time to treatment failure in patients with melanoma treated with ipilimumab at Memorial Sloan Kettering Cancer Center. J Clin Oncol. 2015;33:3193-8. https://doi.org/10.1200/JCO.2015. 60.8448 .

23. Bowyer S, Prithviraj P, Lorigan P, Larkin J, McArthur G, Atkinson $\mathrm{V}$, et al. Efficacy and toxicity of treatment with the anti-CTLA-4 antibody ipilimumab in patients with metastatic melanoma after prior anti-PD-1 therapy. Br J Cancer. 2016;114:1084-9.

24. Eggermont AMM, Chiarion-Sileni V, Grob JJ, Dummer $\mathrm{R}$, Wolchok JD, Schmidt H, et al. Prolonged survival in stage III melanoma with ipilimumab adjuvant therapy. N Engl J Med. 2016;375:1845-55. https://doi.org/10. 1056/NEJMoa1611299.

25. Bertrand A, Kostine M, Barnetche $\mathrm{T}$, et al. Review immune related adverse events associated with antiCTLA-4 antibodies: systematic review and meta-analysis. BMC Med. 2015;13:211.

26. Maughan BL, Bailey E, Gill DM, Agarwal N. Incidence of immune-related adverse events with program death receptor-1- and program death receptor-1 ligand-directed therapies in genitourinary cancers. Front Oncol. 2017;7:56. https://doi.org/10.3389/fonc.2017.00056.

27. Villadolid J, Amin A. Immune checkpoint inhibitors in clinical practice: update on management of immunerelated toxicities. Transl Lung Cancer Res. 2015;4(5):560-75.

28. Topalian SL, et al. Safety, activity, and immune correlates of anti-PD-1 antibody in cancer. N Engl J Med. 2012;366(26):2443-54. https://doi.org/10.1056/ NEJMoa1200690.

29. Larkin J, Chiarion-Sileni V, Gonzalez R, Grob JJ, Cowey $\mathrm{CL}$, Lao CD, et al. Combined nivolumab and ipilimumab or monotherapy in untreated melanoma. N Engl J Med. 2015;373:23-34. https://doi.org/10. 1056/NEJMoa1504030.

30. Weinstock C, Skhozin S, Suzman D, et al. United States Food and Drug Administration approval summary: atezolizumab for metastatic non-small cell lyng cancer. Clin Cancer Res. 2017;23(16):4534-45.

31. Slovin SF, Higano CS, Hamid O, et al. Ipilimumab alone or in combination with radiotherapy win metastatic castration-resistant prostate cancer: results from an open-label, multicenter phase I/II study. Ann Oncol. 2013;24:1813-31.

32. Kwon ED, Drake CG, Scher HI, Fizazi K, Bossi A, van den Eertwegh A, et al. Ipilimumab versus placebo after radiotherapy in patients with metastatic castrationresistant prostate cancer that had progressed after docetaxel chemotherapy (CA184-043): a multicenter, randomized, double-blind, phase 3 trial. Lancet Oncol. 2014;15:700-12.

33. Marthey L, Mateus C, Mussini C, Nachury M, Nancey S, Grange F, et al. Cancer immunotherapy with antiCTLA-4 monoclonal antibodies induces an inflammatory bowel disease. J Crohns Colitis. 2016;10:395-401. https://doi.org/10.1093/ecco-jcc/jjv227

This study describes CTLA-4-induced enterocolitis and describes similarities between this entity and inflammatory bowel disease and that management should be similar.

34. Herbst RS, Baas P, Kim D. Pembrolizumab versus docetaxel for previously treated PD-L1 positive, advanced non-small-cell lung cancer (KEYNOTE-010): a randomized controlled trial. Lancet. 2016;387:1540-50. https://doi.org/10.1016/S0140-6736(15)01281-7. 
35. Berger R, Rotem-Yehudar R, Slama G, Landes S, Kneller A, Leiba M, et al. Phase I safety and pharmacokinetic study of CT-011, a humanized antibody interacting with PD-1, in patients with advanced hematologic malignancies. Clin Cancer Res. 2008;14:3044-51. https://doi.org/10.1158/1078-0432.CCR-07-4079.

36. Hodi FS, O'Day SJ, McDermott DF, Weber RW, Sosman JA, Haanen JB, et al. Improved survival with ipilimumab in patients with metastatic melanoma. $\mathrm{N}$ Engl J Med. 2010;363:711-23. https://doi.org/10. 1056/NEJMoa1003466.

37. Ibrahim RA, Berman DM, DePril V, et al. Ipilimumab safety profile: summary of findings from completed trials in advanced melanoma. J Clin Oncol.

2011;29(15_suppl):8583.

38. Geukes Foppen MH, Rozeman EA, van Wilpe S, et al. Immune checkpoint inhibition-related colitis: symptoms, endoscopic features, histology and response to management. ESMO Open. 2018;3:e000278.

39. Gupta A, De Felice KM, Loftus EV Jr, et al. Systematic review: colitis associated with anti-CTLA-4 therapy. Aliment Pharmacol Ther. 2015;42(4):406-17. https:// doi.org/10.1111/apt.13281.

40. Wang DY, Ye F, Zhao S, Johnson DB. Incidence of immune checkpoint inhibitor-related colitis in solid tumor patients: a systematic review and meta-analysis. Oncoimmunology. 2017;6(10):e1344805. https://doi. org/10.1080/2162402X.2017.1344805.

41. Dougan M. Checkpoint blockade toxicity and immune homeostasis in the gastrointestinal tract. Front Immunol. 2017. https://doi.org/10.3389/fimmu. 2017.01547

42. Wolchok JD, Neyns B, Linette G, Negrier S, Lutzky J, Thomas L, et al. Ipilimumab monotherapy in patient $s$ with pretreated advanced melanoma: a randomized, double-blind, multicenter phase 2, dose-ranging study. Lancet Oncol. 2010;11:155-64.

43. Brahmer J, Reckamp KL, Baas P, Crinò L, Eberhardt WEE, Poddubskaya E, et al. Nivolumab versus docetaxel in advanced squamous-cell non-small-cell lung cancer. N Engl J Med. 2015;373:123-35. https://doi. org/10.1056/NEJMoa1504627.

44. Robert C, Schacter J, Long GV, et al. Pembrolizumab versus ipilimumab in advanced melanoma. $\mathrm{N}$ Engl J Med. 2015;372:2521-32.

45. Ananthakrishnan AN, Higuchi LM, Huang ES, et al. Aspirin, non-steroidal anti-inflammatory drug use, and risk for Crohn's disease and ulcerative colitis: a cohort study. Ann Intern Med. 2012;156:350-9.

46. Menzies AM, Johnson DB, Ramanujam S, Atkinson VG, Wong ANM, Park JJ, et al. Anti-PD-1 therapy in patients with advanced melanoma and preexisting autoimmune disorders or major toxicity with ipilimumab. Ann Oncol. 2017;28:368-76.

47. Johnson DB, Sullivan RJ, Ott PA, Carlino MS, Khushalani NI, Ye F, et al. Ipilimumab therapy in patients with advanced melanoma and preexisting autoimmune disorders. JAMA Oncol. 2016;2(2):234-40. https://doi.org/10.1001/jamaoncol.2015.4368.
48. Postow MA, Chesney J, Pavlick AC, Robert C, Grossmann K, McDermott D, et al. Nivolumab and ipilimumab versus ipilimumab in untreated melanoma. N Engl J Med. 2015;372:2006-17. https://doi.org/ 10.1056/NEJMoa1414428.

49. Wolchok JD, Chiarion-Sileni V, Gozalez R, et al. Overall survival with combined nivolumab and ipilimumab in advanced melanoma. N Engl J Med. 2017;377:1345-56. https://doi.org/10.1056/ NEJMoa1709684.

50. Hodi FS, Chesney J, Pavlick AC, Robert C, Grossmann $\mathrm{KF}, \mathrm{McDermott} \mathrm{D}$, et al. Combined nivolumab and ipilimumab versus ipilimumab alone in patients with advanced melanoma: 2-year overall survival outcomes in a multicentre, randomised, controlled, phase 2 trial. Lancet Oncol. 2016;17:1558-68.

51. Sznol M, Ferrucci PF, Hogg D, et al. Pooled analysis safety profile of nivolumab and ipilimumab combination therapy in patients with advanced melanoma. J Clin Oncol. 2017;35(34):3815-22. https://doi.org/10. 1200/JCO.2016.72.1167.

52. Danlos FX, Pagès C, Roux J, et al. Atypical severe immune-related adverse effects resulting from sequenced immunotherapy in melanoma. Melanoma Res. 2015;25:178-9.

53. Khoja L, Butler MO, Chappell MA, et al. Increased treatment-related toxicity subsequent to an anti-PD-1 agent. Curr Oncol. 2015;22(4):320-2.

54. Robert C, Thomas L, Bondarenko I, O'Day S, Weber J, Garbe $\mathrm{C}$, et al. Ipilimumab plus dacarbazine for previously untreated metastatic melanoma. N Engl J Med. 2011;364:2517-26. https://doi.org/10.1056/ NEJMoa1104621.

55. Lord JD, Hackman RC, Moklebust A, et al. Refractory colitis following anti-CTLA4 antibody therapy: analysis of mucosal FOXP3+ T cells. Dig Dis Sci. 2010;55:1396405.

56. Gentile NM, D'Souza A, Fujii LL, Wu TT, Murray JA. Association between ipilimumab and celiac disease. Mayo Clin Proc. 2013;88:414-7. https://doi.org/10. 1016/j.mayocp.2013.01.015.

57. Abdel-Wahab N, Shah M, Suarez-Almazor ME. Adverse events associated with immune checkpoint blockade in patients with cancer: a systematic review of case reports. PLoS One. 2016;11:e0160221. https://doi.org/ 10.1371/journal.pone.0160221.s004.

58. Kobayashi M, Yamaguchi O, Nagata K, Nonaka K, Ryozawa S. Acute hemorrhagic gastritis after nivolumab treatment. Gastrointest Endosc. 2017;86(5):915-6. https://doi.org/10.1016/j.gie. 2017.04.033.

59. Verschuren EC, van den Eertwegh AJ, Wonders J, Slangen RM, van Delft F, van Bodegraven A, et al. Clinical, endoscopic, and histologic characteristics of ipilimumab-associated colitis. Clin Gastroenterol Hepatol. 2016;14:836-42.

60. Robert C, Long GV, Brady B, Dutriaux C, Maio M, Mortier $\mathrm{L}$, et al. Nivolumab in previously untreated melanoma without BRAF mutation. N Engl J Med. 
2015;372:320-30. https://doi.org/10.1056/ NEJMoa1412082.

61. Garon EB, Rizvi NA, Hui R, Leighl N, Balmanoukian AS, Eder JP, et al. Pembrolizumab for the treatment of non-small-cell lung cancer. N Engl J Med.

2015;372:2018-28. https://doi.org/10.1056/

NEJMoa1501824.

62. Bellmunt J, de Wit R, Vaughn DJ, Fradet Y, Lee J-L, Fong $\mathrm{L}$, et al. Pembrolizumab as second-line therapy for advanced urothelial carcinoma. N Engl J Med. 2017;376:1015-26. https://doi.org/10.1056/ NEJMoa1613683.

63. Reck M, Rodríguez-Abreu D, Robinson AG, Hui R, Csőszi T, Fülöp A, et al. Pembrolizumab versus chemotherapy for PD-L1-positive non-small-cell lung cancer. N Engl J Med. 2016;375:1823-33. https://doi. org/10.1056/NEJMoa1606774.

64. Abu-Sbeih H, Ali FS, Alsaadi D, et al. Outcomes of vedolizumab therapy in patients with immune checkpoint inhibitor-induced colitis: a multi-center study. J Immunother Cancer. 2018;6:142

This study demonstrates that vedolizumab can be a viable alternative to infliximab in the treatment of certain patients with immune-mediated colitis.

65. Berman D, Parker SM, Siegel J, et al. Blockade of cytotoxic T-lymphocyte antigen-4 by ipilimumab results in dysregulation of gastrointestinal immunity in patients with advanced melanoma. Cancer Immun. 2010;10:11.

66. Chatzikonstantinou M, Konstantopoulos P, Stergiopoulos S, et al. Calprotectin as a diagnostic tool for inflammatory bowel diseases (review). Biomed Rep. 2016;5:403-7. https://doi.org/10.3892/br.2016.751.

67. Abej E, El-Matary W, Singh H, Bernstein CN. The utility of fecal calprotectin in the real-world clinical care of patients with inflammatory bowel disease. Canadian J Gastroenterol Hepatol. 2016: 6 pages:2483261.

68. Kim KW, Ramaiya NH, Krajewski KM, Shinagare AB, Howard SA, Jagannathan JP, et al. Ipilimumabassociated colitis: CT findings. AJR Am J Roentgenol. 2013;200:W468-74. https://doi.org/10.2214/AJR.12. 9751.

69. Friedman CF, Proverbs-Singh TA, Postow MA. Treatment of the immune-related adverse effects of immune checkpoint inhibitors: a review. JAMA Oncol.

2016;2:1346-53. https://doi.org/10.1001/jamaoncol. 2016.1051.

70. Johnson DH, Zobniw CM, Trinh VA, et al. Infliximab associated with faster symptom resolution compared with corticosteroids alone for the management of immune-related enterocolitis. J Immunother Cancer. 2018;6(1):103. https://doi.org/10.1186/s40425-0180412-0.

71. Abu-Sbeih H, Ali FS, Wang X, et al. Early introduction of selective immunosuppressive therapy associated with favorable clinical outcomes in patients with immune checkpoint inhibitor-induced colitis. J Immunother Cancer. 2019;7:93. https://doi.org/10. 1186/s40425-019-0577-1
This study demonstrates that early intervention with biologic therapy improved clinical outcomes in patients with immunemediated colitis.

72. Abu-Sbeih H, Tang T, Ali FS, et al. The impact of immune checkpoint inhibitor-related adverse events and their immunosuppressive treatment of patients outcomes. J Immunother Precis Oncol. 2018;1:7-18.

73.• Clinical management algorithms - the University of Texas MD Anderson Cancer Center. Evaluation and management of suspected immune-mediated colitis/ diarrhea. https://www.Mdanderson.Org/content/dam/ mdanderson/documents/for-physicians/algorithms/ clinical-management/clinmanagement-immunemediated-colitis-web-algorithm.Pdf. 2018.

These guidelines provide step-by-step management for patients with immune-mediated colitis.

74. Hseih AH, Ferman M, Brown MP, et al. Vedolizumab: a novel treatment for ipilimumab-induced colitis. BMJ Case Rep. 2016. https://doi.org/10.1136/bcr-2016216641.

75. Bergqvist V, Hertervig E, Gedeon P, Kopljar M, Griph $\mathrm{H}$, Kinhult $\mathrm{S}$, et al. Vedolizumab treatment for immune checkpoint inhibitor-induced enterocolitis. Cancer Immunol Immunother. 2017;66:581-92. https://doi. org/10.1007/s00262-017-1962-6.

76. Colombel JF, Sands BE, Rutgeerts P, Sandborn W, Danese S, D'Haens G, et al. The safety of vedolizumab for ulcerative colitis and Crohn's disease. Gut. 2017;66:839-51.

77. Vetizou M, Pitt JM, Daillere R, et al. Anticancer immunotherapy by CTLA- 4 blockade relies on the gut microbiota. Science. 2015;350:1079-84.

78. Gopalakrishnan V, Spencer CN, Nezi L, Reuben A, Andrews MC, Karpinets TV, et al. Gut microbiome modulates response to anti-pd-1 immunotherapy in melanoma patients. Science. 2018;359:97-103.

79. Dubin K, Callahan MK, Ren B, et al. Intestinal microbiome analyses identify melanoma patients at risk for checkpoint-blockade-induced colitis. Nat Commun. 2016;7:10391. https://doi.org/10.1038/ ncomms10391.

80. Chaput N, Lepage P, Coutzac C, Soularue E, le Roux K, Monot $\mathrm{C}$, et al. Baseline gut microbiota predicts clinical response and colitis in metastatic melanoma patients treated with ipilimumab. Ann Oncol. 2017;28:136879.

81. Wang Y, Wiesnoski D, Helmink B, Gopalakrishnan V, Choi K, DuPont H, et al. Fecal microbiota transplantation for refractory immune checkpoint inhibitorassociated colitis. Nat Med. 2018;24(12):1804-8.

82. Schadendorf D, Wolchok JD, Hodi FS, Chiarion-Sileni V, Gonzalez R, Rutkowski P, et al. Efficacy and safety outcomes in patients with advanced melanoma who discontinued treatment with nivolumab and ipilimumab because of adverse events: a pooled analysis of randomized phase II and III trials. J Clin Oncol. 2017;34:3807-14. https://doi.org/10.1200/JCO.2017. 73.2289. 
83. Amin A, de Pril V, Hamid O, et al. Evaluation of the effect of systemic corticosteroids for the treatment of immune-related adverse events (irAEs) on the development or maintenance of ipilimumab clinical activity. J Clin Oncol. 2009;27(15_suppl):9037-7.

84. Harmankaya K, Erasim C, Koelblinger C, et al. Continuous systemic corticosteroids do not affect the ongoing regression of metastatic melanoma for more than two years following ipilimumab therapy. Med Oncol. 2011;28:1140-4. https://doi.org/10.1007/ s12032-010-9606-0.

85. Baurain JF, Smylie M, Ascierto PA, et al. Outcomes of ipilimumab treatment-related adverse events in patients with metastatic melanoma (MM) who received systemic corticosteroids in a phase 3 trial. J Clin Oncol. 2012;30(15_suppl):8539-9. https://doi.org/10.1200/ jco.2012.30.15_suppl.8539.

86. U.S. Department of Health and Human Services. National Institutes of Health. National Cancer Institute.
Available at: https://ctep.cancer.gov/ protocoldevelopment/electronic_applications/docs/ CTCAE_v5_Quick_Reference_5x7.pdf . Accessed 1 April 2019.

87.• Thompson JA. New NCCN guidelines: recognition and management of immunotherapy-related toxicity. J Natl Compr Cancer Netw. 2018;15:594-6

These are the current National Comprehensive Cancer Network guidelines on management of immune-related adverse events.

\section{Publisher's Note}

Springer Nature remains neutral with regard to jurisdictional claims in published maps and institutional affiliations. 